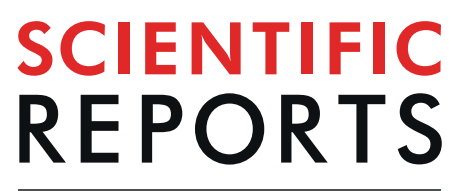

natureresearch

\title{
OPEN Non-thermal plasma inhibits mast cell activation and ameliorates allergic skin inflammatory diseases in NC/Nga mice
}

Received: 11 March 2019

Accepted: 22 July 2019

Published online: 18 September 2019

\author{
Myung-Hoon Lee ${ }^{1,2}$, Yun Sang Lee ${ }^{1}$, Haeng Jun Kim ${ }^{1,2}$, Chang Hak Han ${ }^{1,2}$, Sung Un Kang ${ }^{1}$ \& \\ Chul-Ho Kim ${ }^{1,2}$
}

Non-thermal plasma (NTP) has many functional activities such as, sterilization, wound healing and anti-cancer activity. Despite of its wide spread biomedical application, the effect of NTP on immune cells and allergic response has not been well studied. In this study, we determined whether NTP suppresses mast cell activation, which is important for allergic response, and ameliorates an atopic dermatitis (AD)-like skin inflammatory disease in mice. Exposure to NTP-treated medium during mast cell activation inhibited the expression and production of IL-6, TNF- $\alpha$ and suppressed NF- $\kappa B$ activation. We also investigated whether NTP treatment ameliorates house dust mite (HDM)-induced AD-like skin inflammation in mice. NTP treatment inhibited increases in epidermal thickness and recruitment of mast cells and eosinophils, which are important cell types in AD pathogenesis. In addition, Th2 cell differentiation was induced by application of HDM and the differentiation was also inhibited in the draining lymph node of NTP-treated mice. Finally, the expression of AD-related cytokines and chemokines was also decreased in NTP-treated mice. Taken together, these results suggest that NTP might be useful in the treatment of allergic skin diseases, such as AD.

Atopic dermatitis (AD) is a common allergic skin disease, characterized by mast cell activation, eosinophilia, overexpression of cytokines and epithelial hyperplasia ${ }^{1,2}$. Although the etiology of AD is complex, many studies suggest that immune cells are involved in the pathogenesis of $\mathrm{AD}$ and uncontrolled immune response is one of the main causes of $\mathrm{AD}^{3}$. For example, in the acute phase of $\mathrm{AD}$, Th2 cell percentage increases, and $\mathrm{AD}$ skin lesions express higher levels of Th2 cytokines and chemokines compared to normal skin ${ }^{4,5}$. In addition, the activation and infiltration of mast cells and eosinophils are also critical for $\mathrm{AD}^{6}$. By contrast, Th1 cells and their cytokines play a role in the chronic phase of $\mathrm{AD}^{7}$. Several treatments for $\mathrm{AD}$, such as glucocorticoids, calcineurin inhibitors, phototherapy, and immunosuppressors (cyclosporine A), have been used ${ }^{8}$. However, these drugs and therapies cause many side effects including ulcers, thin skin, diabetes, depression, and slow-wound healing ${ }^{9}$. Therefore, the development of new treatments for $\mathrm{AD}$ without side effects imperative.

Plasma is referred to as the fourth state of matter and is composed of cations, anions, electrons and reactive species. During the last few decades, the field of plasma medicine has grown rapidly. Several studies show that plasma regulates various effects, such as anti-cancer ${ }^{10-13}$, anti-inflammation ${ }^{14}$, sterilization ${ }^{15}$, and tooth-bleaching effects $^{16}$. Recently, we demonstrated that non-thermal plasma (NTP) treatment induces cancer cell death via AKT degradation ${ }^{17}$, promotes muscle regeneration in mice ${ }^{18}$, accelerates wound healing ${ }^{19}$, and inhibits psoriasis-like skin inflammation in mice ${ }^{20}$. Thus, plasma medicine is emerging in the biomedical field, and researchers are investigating whether plasma can be used for the treatment of various diseases. However, the anti-allergic effect of NTP is not well studied.

In this study, we investigated whether NTP inhibited mast cell activation and house dust mite (HDM)-induced AD-like skin inflammation in NC/Nga mice. Our results show that NTP treatment inhibited HDM-induced $\mathrm{AD}$-like skin inflammation in NC/Nga mice. In addition, we demonstrated the inhibitory effect of liquid type

${ }^{1}$ Department of Otolaryngology, School of Medicine, Ajou University, Suwon, Republic of Korea. ${ }^{2}$ Department of Molecular Science and Technology, Ajou University, Suwon, Republic of Korea. Myung-Hoon Lee and Yun Sang Lee contributed equally. Correspondence and requests for materials should be addressed to C.-H.K. (email: ostium@ ajou.ac.kr) 
plasma (LTP) on mast cell activation and the inflammation of keratinocytes, suggesting that the anti-allergic effect of NTP might at least partially result from the suppression of mast cell and keratinocyte activation.

\section{Results}

LTP treatment inhibits mast cell activation. We investigated whether plasma treatment inhibits mast cell activation, which is involved in an allergic response. To generate mouse primary mast cells, lineage-negative cells were isolated from bone marrow and cultured with mIL-3 and mSCF for 6 weeks (Fig. 1a) as described in Materials and Methods. LTP was made as shown in Fig. $1 \mathrm{~b}$ and the mast cells were activated with PMA and A23187 with or without plasma-treated medium. As shown in Fig. 1c, NF- $\kappa B$ is activated in activated mast cells and LTP treatment inhibited the NF- $\kappa$ B activation. Activated mast cells also expressed increased proinflammatory cytokines such as TNF- $\alpha$, IL- 6 and IL-13, however, LTP treatment inhibited the increased levels of TNF- $\alpha$, IL-6 and IL-13 expression (Fig. 1d-f). Consistently, the results of ELISA also showed that LTP treatment decreased the secretion of TNF- $\alpha$, IL-6 and IL-13 (Fig. 1g-i). These results suggest that LTP treatment decreased the cytokine expression via inhibition of NF- $\kappa$ B activation and plasma treatment might inhibit the allergic response.

NTP treatment ameliorate HDM-induced AD-like skin inflammation in mice. LTP treatment suppressed mast cell activation, suggesting that plasma could have anti-allergic effect. Thus, we investigated whether NTP could suppress AD-like skin inflammation in mice. After shaving back hair, a cream containing HDM (Biostir) was applied on NC/Nga mice every 4 days for 24 days, and NTP was treated every other day between day 9 and day 25. The mice were sacrificed on day 26 for analysis (Fig. 2a,b). Application of HDM induced AD-like skin inflammation in NC/Nga mouse skin and NTP treatment ameliorated the skin inflammation (Fig. 2c). Hematoxylin and Eosin (H\&E) staining also revealed an increased epidermal thickness and immune cell infiltration in HDM-applied skin, while NTP treatment decreased the epidermal thickness and immune cell infiltration (Fig. 2d,e). We determined the number of eosinophils and mast cells among the infiltrated immune cells in the dermis because the cells are involved in the pathogenesis of AD. As shown in Fig. 3, exposure to HDM increased the infiltration of eosinophils (Fig. 3a,b) and mast cells (Fig. 3c,d). Meanwhile, NTP treatment suppressed the HDM-induced eosinophil and mast cell infiltration into the dermis. NTP treatment without HDM stimulation did not affect eosinophil and mast cell infiltration, suggesting that NTP treatment has no affect in normal skin.

NTP treatment inhibits Th2 cell differentiation and AD-related gene expression in mice. Th2 cells are one of the major cell types involved in AD pathogenesis. Thus, we determined whether NTP treatment affected Th2 cell differentiation in mice. As expected, the proportion of Th2 cells increased in the draining lymph node of HDM treated- mice (Fig. 4c,e). However, NTP treatment decreased the Th2 cell percentage (Fig. 4d,e), suggesting that NTP treatment inhibits Th2 cell differentiation. Th2 cell percentage in NTP-treated mice without HDM stimulation was not changed compared with that of NTP-untreated mice (Fig. 4a,b,e), implying that NTP treatment might not regulate unstimulated immune cells in normal mice. Consistently, treatment of LTP during Th2 cell differentiation attenuated the differentiation from naïve CD4 T cells to Th2 cells in vitro (Supplementary Fig. 1). These results imply that NTP treatment might inhibit Th2 cell differentiation

We also determined the $\mathrm{AD}$-related cytokine and chemokine expression in mouse skin tissue. The levels of RNA expression of TSLP (Fig. 5a) and CCL17 (Fig. 5b) were increased in AD-induced mouse skin and were reduced by NTP treatment. In addition, using IHC analysis we confirmed that the production of TSLP, which is an important cytokine for AD pathogenesis, was also suppressed. As shown in Fig. 5c, the production of TSLP increased in HDM-applied mouse skin compared with that of HDM/NTP-treated mouse skin, which is consistent with real-time PCR results. The intensity of the TSLP signal was measured using ImageJ and shown as a graph (Fig. 5d).

LTP treatment inhibits the inflammatory response in HaCaT. To confirm the anti-allergic inflammatory effect in vitro, we stimulated HaCaT, a keratinocyte cell line, with IL-4 and/or LTP and measured the level of STAT6 activation. The IL-4-stimulated group showed an increase in STAT6 activation. By contrast, the IL-4/LTP-treated group showed a decrease in STAT6 activation compared with IL-4 only stimulated group (Fig. 6a). In addition, LTP treatment inhibited IL-4-induced CCL26 expression, which mediated eosinophil recruitment (Fig. 6b). Expression of pro-inflammatory cytokines, such as TNF- $\alpha$ and IFN- $\gamma$, was also increased and stimulated other cells in chronic allergic responses. Thus, we determined whether LTP treatment inhibited TNF- $\alpha / \mathrm{IFN}-\gamma$-induced NF- $\kappa \mathrm{B}$ activation and pro-inflammatory cytokine expression in HaCaT. Stimulation with TNF- $\alpha$ and IFN- $\gamma$ induced NF- $\kappa \mathrm{B}$ activation and increased the expression of pro-inflammatory cytokines, such as IL-8, IL-6, and TNF- $\alpha$. However, LTP treatment suppressed the level of NF- $\kappa$ B activation (Fig. 6c) and pro-inflammatory cytokine expression (Fig. 6d-f) suggesting that plasma treatment might inhibit not only acute, but also chronic allergic responses.

\section{Discussion}

Plasma medicine is an emerging field combining plasma physics and biomedical science for therapeutic application. Since the first clinical trials using NTP in 2010 to reduce bacteria in chronic wounds ${ }^{21}$, many experiments were performed and showed various effects including anti-cancer ${ }^{22}$, muscle regeneration ${ }^{18}$, wound healing ${ }^{23}$, and anti-inflammatory effect ${ }^{20}$. However, anti-allergic effect of plasma is not well-studied. In this study, we investigated anti-allergic effect of plasma in mice using mouse model of AD.

$\mathrm{AD}$ is a common allergic inflammatory skin disease and one of the major public health issues worldwide, with increasing incidence ${ }^{24}$. The etiology of AD involves immune system dysfunction, environmental exposures, and genetics. However, appropriate treatment has yet to be developed and the current treatments for AD only inhibit the disease symptoms without curing the disease $\mathrm{e}^{25}$. Furthermore, current therapies have various side effects ${ }^{26,27}$. Thus, development of more effective therapies without side effects is necessary for the treatment of AD patients. 
a
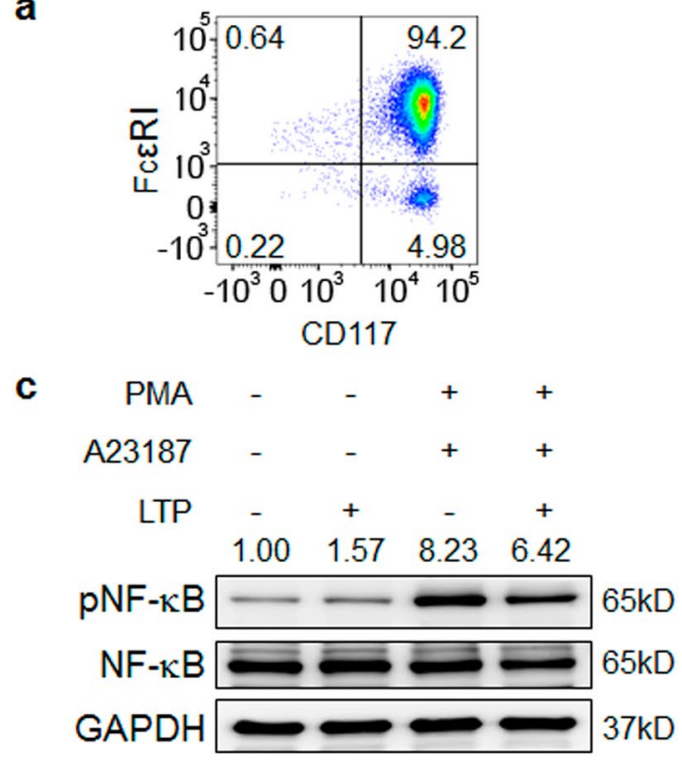

b

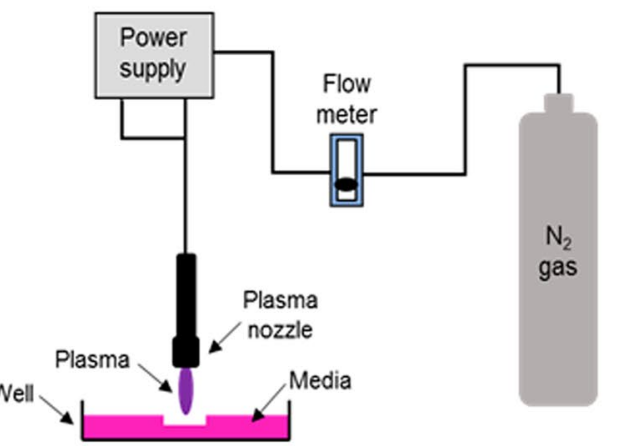

d

TNF- $\alpha$

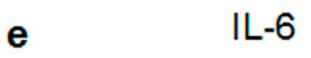

$\mathbf{f}$

IL-13
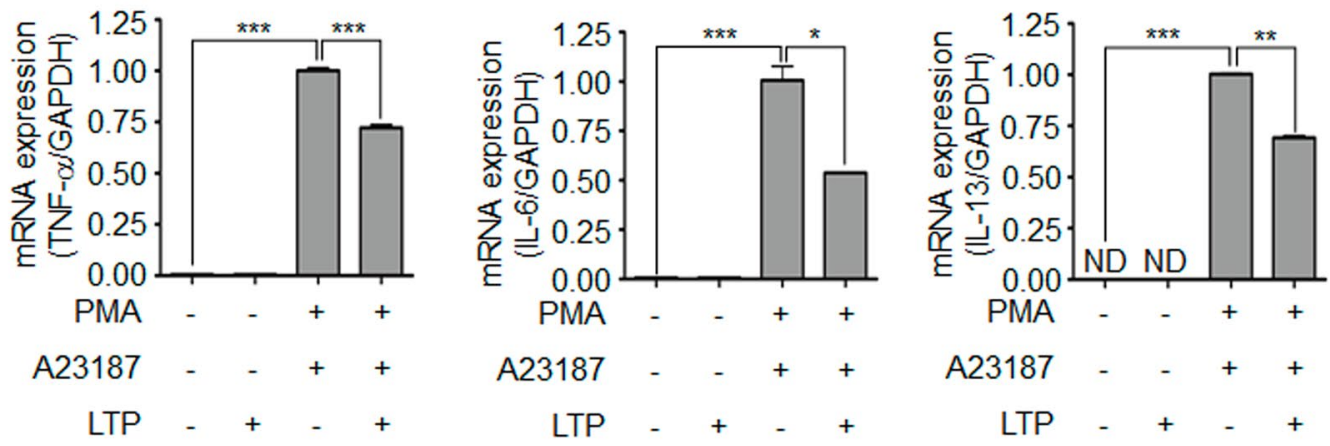

g

TNF- $\alpha$

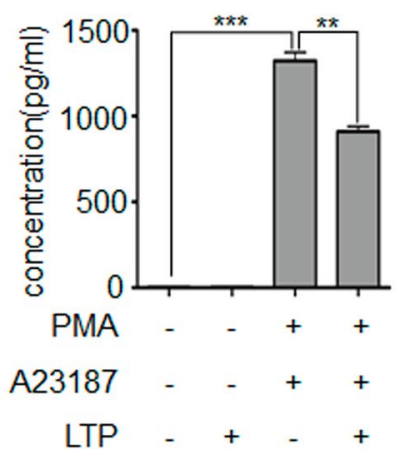

h

IL-6

i

IL-13
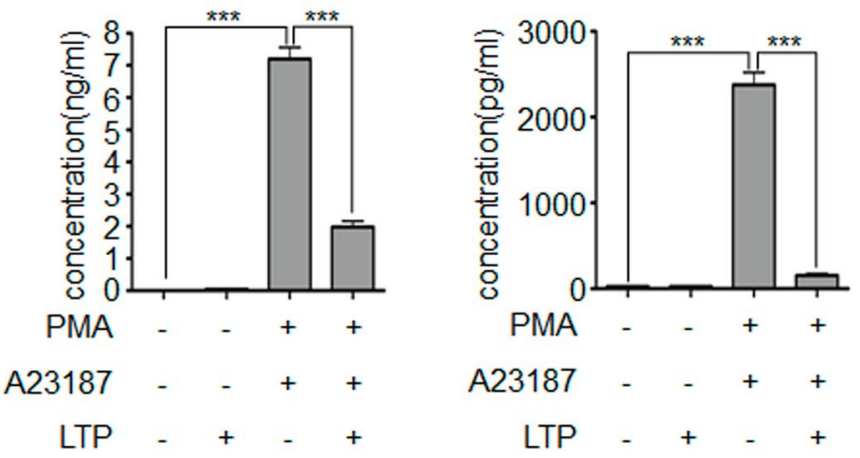

Figure 1. LTP inhibits mast cell activation. (a) Mast cells were generated from mouse bone-marrow derived lineage negative cells by culture with mIL-3 and mSCF for 8 weeks. The percentage of mast cells exceed $94 \%$. (b) A schematic diagram showing the generation of LTP. Non-thermal plasma was treated for $60 \mathrm{sec}$ per $\mathrm{ml}$ and used for in vitro experiments. (c) All Western blotting experiments were performed under the same condition. LTP treatment inhibits NF- $\kappa B$ activation in PMA/A23187-stimulated mast cells. LTP treatment inhibits (d) TNF- $\alpha$, (e) IL-6, and (f) IL-13 expression in PMA/A23187-stimulated mast cells. LTP treatment reduced the secretion of (g) TNF- $\alpha$, (h) IL-6, and (i) IL-13 by activated mast cells. $* P<0.05, * * P<0.01, * * * P<0.001$.

$\mathrm{HDM}$ is one of the most well-known allergens inducing $\mathrm{AD}^{28}$. Thus, we used HDM to induce AD-like skin inflammation in NC/Nga mice. Repeated application of HDM to the shaved skin of the mice induced symptoms of AD-like allergic skin inflammation, such as increased epidermal thickness, upregulation of cytokine and 
a

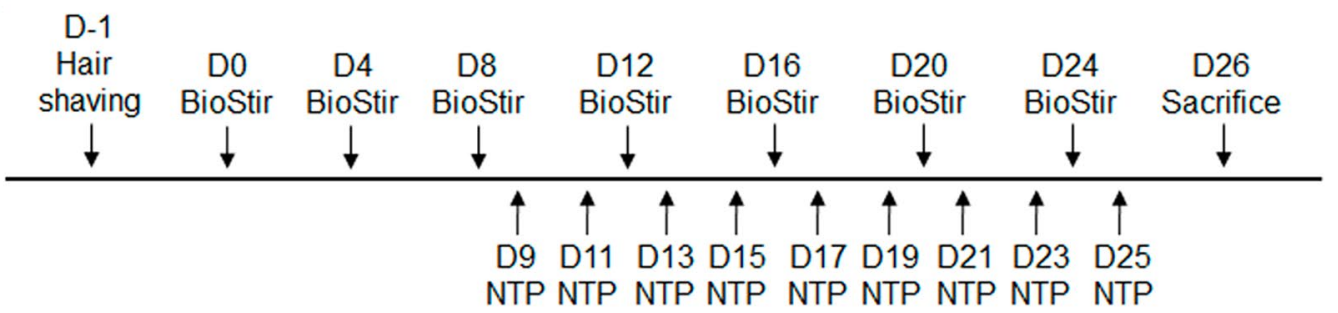

b

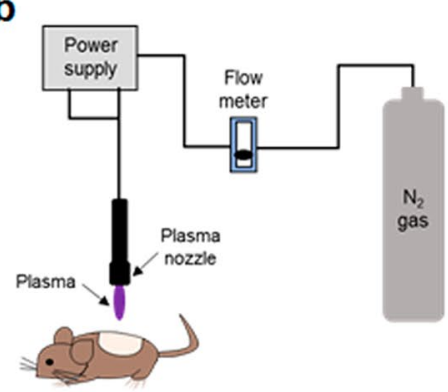

C

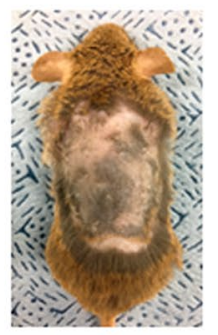

Non-treatment

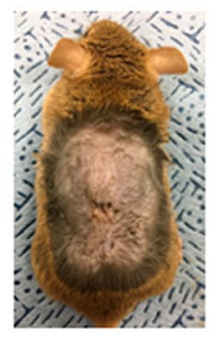

NTP only

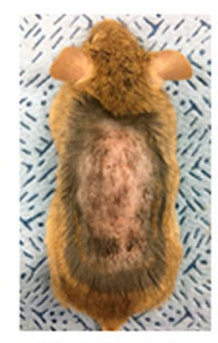

Biostir only

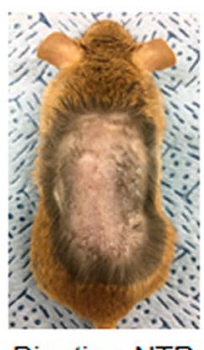

Biostir + NTP

d

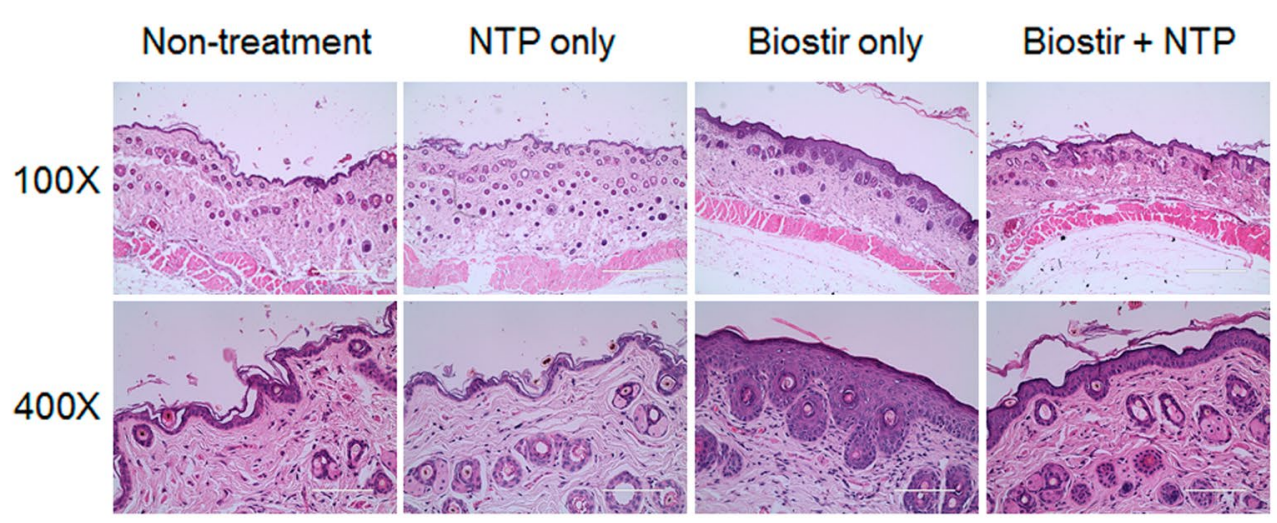

e

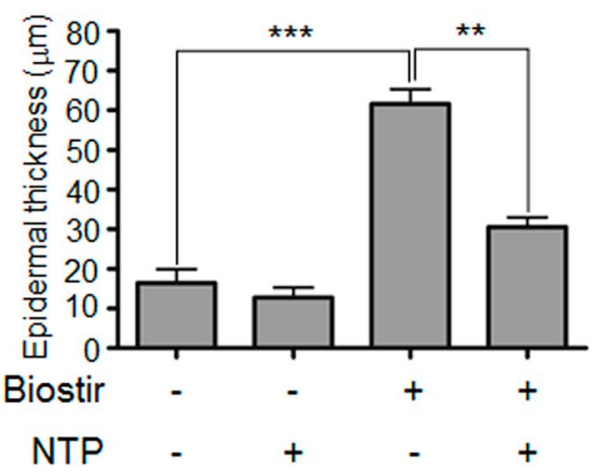

Figure 2. NTP treatment ameliorates HDM-induced AD-like skin inflammation in NC/Nga mice. (a) The experimental scheme for induction of HDM-induced AD-like skin inflammation and NTP treatment. (b) A schematic diagram describing treatment of mice with the NTP- producing machine. (c) NTP treatment inhibited AD-like skin inflammation in NC/Nga mice. (d) H\&E staining of mouse back skin (e) NTP treatment reduced epidermal thickness. $* * P<0.01, * * * P<0.001$. Bar $=400 \mu \mathrm{m}(100 \times), 100 \mu \mathrm{m}(400 \times)$.

chemokine expression, and immune cell infiltration into the dermis ${ }^{29-31}$. Indeed, repeated application of HDM in $\mathrm{NC}$ /Nga mice induced AD-like skin inflammation, and $\mathrm{N}_{2}$-based NTP treatment ameliorated the AD-like allergic skin inflammation. NTP treatment decreased epidermal thickness (Fig. 2) and inhibited skin cell proliferation (Supplementary Fig. 6). Immune cell infiltration into skin tissue was also suppressed by LTP treatment (Fig. 2).

In vitro experiments are also showed that plasma treatment could inhibits skin inflammatory response in keratinocytes. Plasma treatment inhibits TNF- $\alpha /$ IFN- $\gamma$-induced NF- $\kappa B$ activation and translocation into the nucleus (Fig. $6 \mathrm{c}$ and Supplementary Fig. 5). IL-4 is an important cytokine for AD pathogenesis. Thus, we 
a
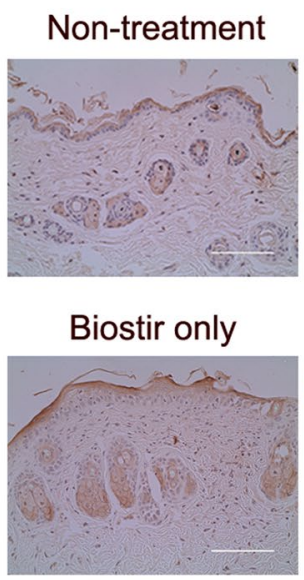

C
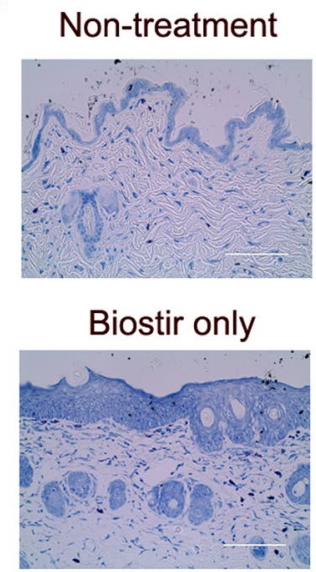

NTP only

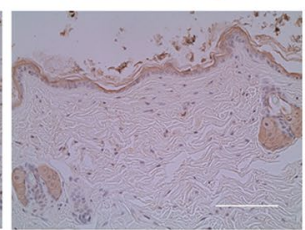

Biostir + NTP

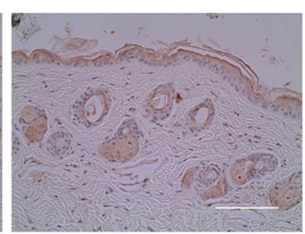

NTP only

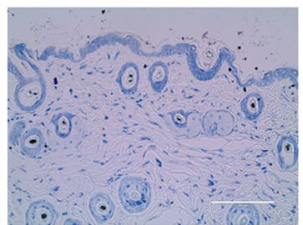

Biostir + NTP

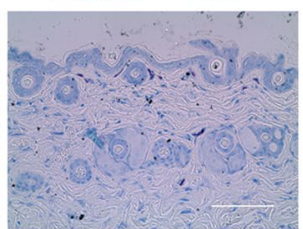

b

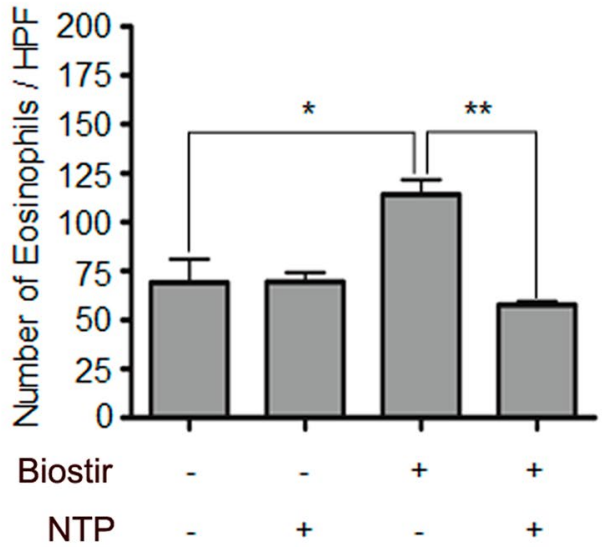

d

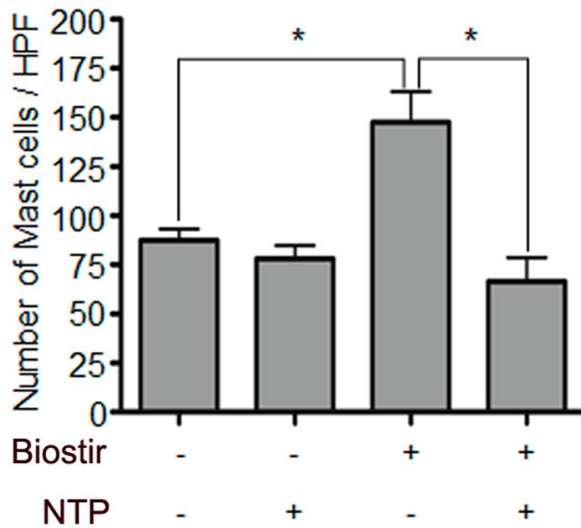

Figure 3. NTP treatment inhibits immune cell infiltration in the mouse skin. (a) NTP treatment inhibited eosinophil infiltration in HDM-induced AD-like mouse skin. (b) The number of infiltrated eosinophils is indicated as a bar graph. (c) NTP treatment inhibited mast cell infiltration in HDM-induced AD-like mouse skin. (d) The number of infiltrated mast cells is represented as a bar graph. $* P<0.05$, $* * P<0.01$. Bar $=100 \mu \mathrm{m}$.

determined whether plasma treatment can suppress IL-4 signaling. Plasma treatment did inhibit IL-4-mediated STAT6 activation (Fig. 6a). In addition, plasma treatment in keratinocytes also suppressed STAT1 and STAT3 activation, which are involved in chronic allergic inflammation and autoimmune diseases, respectively, (Supplementary Fig. 9). He plasma can induce STAT1 and STAT3 activation ${ }^{32}$. However, in this study, we demonstrated that $\mathrm{N}_{2}$ plasma treatment inhibits cytokine-induced STAT1 and STAT3 activation (Supplementary Fig. 9). These results imply that the carrier gas might be important for plasma properties and that $\mathrm{N}_{2}$ plasma can inhibit STAT signaling, which is involved in inflammatory diseases.

We also investigated the effect of $\mathrm{N}_{2}$ plasma on ROS generation in keratinocytes because air plasma treatment increases ROS levels in cancer cells and eventually promotes cancer cell death ${ }^{33}$. In our study, $\mathrm{N}_{2}$ plasma treatment does not induce ROS generation in keratinocytes (Supplementary Fig. 7). The results imply that non-cancer cells may be able to regulate ROS levels after plasma treatment, and the results coincide with a previous report ${ }^{34}$. Furthermore, plasma treatment did not induce apoptosis in keratinocytes, although plasma can induce cell death in cancer cells ${ }^{33}$. Thus, we investigated whether $\mathrm{N}_{2}$ plasma can also induce apoptosis. TUNEL assay and western blot experiments showed that $\mathrm{N}_{2}$ plasma treatment did not induce apoptosis in keratinocytes (Supplementary Fig. 8). Taken together, our results suggest that $\mathrm{N}_{2}$ plasma inhibits inflammatory responses without cell death in keratinocytes.

A recent study demonstrated that Ar-based plasma inhibited DNCB-induced AD-like skin inflammation. However, the results showed that Ar-based plasma treatment was not effective in decreasing epidermal thickness $^{35}$. By contrast, our results showed that treatment with N2-based NTP for AD-like skin inflammation reduced the epidermal thickness along with the inhibition of other allergic responses in HDM-induced AD-like skin inflammation in mice. To figure out the best condition for the anti-allergic effect of non-thermal plasma, more experiments with various plasmas generated from many different conditions should be performed.

In this study, we demonstrated that NTP can ameliorate AD-like skin inflammation in a mouse model of AD induced with HDM in NC/Nga mice. These results might suggest novel applications of NTP for the treatment of $\mathrm{AD}$ and other allergic diseases. 

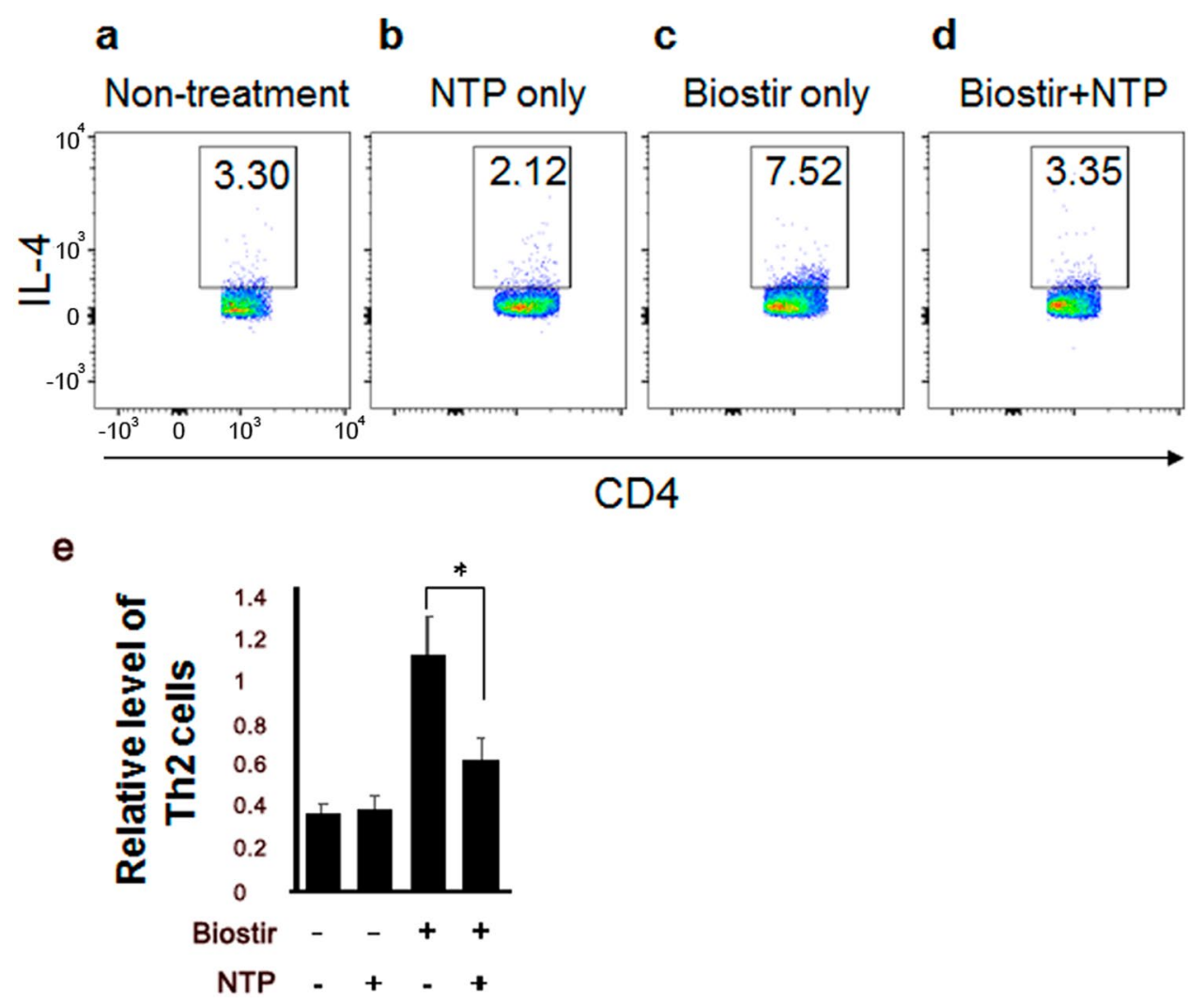

Figure 4. NTP treatment inhibited Th2 cell differentiation in vivo. Th2 cell differentiation in the draining lymph node in (a) non-treated and (b) NTP only-treated mice was similar. (c) HDM (Biostir)-induced Th2 cell differentiation in the mouse draining lymph node. (d) NTP treatment inhibited HDM-induced Th2 cell differentiation. (e) The Relative levels of Th2 cells are shown as a bar graph from four mice. $* P<0.05$, $* * P<0.01$.

\section{Materials and Methods \\ $\mathrm{N}_{2}$ plasma device. The $\mathrm{N}_{2}$ plasma device and the plasma generated from the device were described previ- ously ${ }^{18}$. Briefly, $\mathrm{N}_{2}$ gas was used as a carrier gas of non-thermal atmospheric plasma. The gas flow rate, the input voltage, and the frequency were maintained at $10 \mathrm{~L} / \mathrm{min}, 15 \mathrm{kV}_{\mathrm{p}-\mathrm{p}}$, and $15 \mathrm{kHz}$, respectively. The optical emission spectra and current-voltage profile are shown in Supplementary Fig. 3. Plasma parameter, such as gas tempera- ture, electron temperature and electron density are shown in Supplementary Fig. 4. We determined the presence of $\mathrm{NO}_{2}$ in the plasma-treated solution using a nitric oxide assay kit (Invitrogen) according to the manufacturer's suggested procedures. Exposure of media to plasma for 1 min generated $60 \mu \mathrm{M}$ of $\mathrm{NO}_{2}$.}

LTP generation and treatment. LTP was generated by the treatment of non-thermal $\mathrm{N}_{2}$ plasma into culture media (RPMI 1640 or DMEM) for $60 \mathrm{sec}$ per $\mathrm{ml}$ at a $2 \mathrm{~cm}$ distant from the media (Fig. 6a). Cells were treated with LTP for $6 \mathrm{~h}$ for real-time PCR analysis. HaCaT cells were incubated with LTP for $15 \mathrm{~min}$ for pSTAT6, STAT6, pNF- $\kappa B$ and NF- $\kappa B$ detection.

Cell line and reagents. The immortalized human keratinocyte cell line, HaCaT was grown as monolayer cultures in DMEM (Welgene, Daegu, Korea) supplemented with 10\% fetal bovine serum (Gibco, Carlsbad CA), $1 \%$ penicillin and streptomycin (Gibco). Antibodies for STAT6, p-STAT6, NF- $\kappa$ B, p-NF- $\kappa$ B and GAPDH were purchased from Cell signaling technology (Danvers, MA). Anti-eosinophil peroxidase antibodies were purchased from Santa Cruz biotechnology. All recombinant cytokines were purchased from Peprotech (Rocky Hill, NJ).

RNA isolation, complementary DNA synthesis, and quantitative real-time PCR analysis. Total RNA from cells or mouse skin tissue was isolated using TRIzol reagent (Gibco, Carlsbad, CA). Subsequently, the first strand of cDNA was synthesized with $1 \mu \mathrm{g}$ total RNA using a ReverTra Ace qPCR RT Master Mix (TOYOBO, Japan). The primer sets from Qiagen (Hilden, Germany) were used for real-time PCR analysis and GAPDH transcript was used as an endogenous control. The amplification program consisted of $1 \mathrm{cycle}$ at $95^{\circ} \mathrm{C}$ for $10 \mathrm{~min}$, followed by $45 \mathrm{cycles}$ at $95^{\circ} \mathrm{C}$ for $20 \mathrm{sec}, 55^{\circ} \mathrm{C}$ for $20 \mathrm{sec}$, and 72 for $20 \mathrm{sec}$.

Western blot analysis. Western blot analysis was performed as previously described with minor modification $^{36}$. Cells were lysed with RIPA buffer (Sigma Aldrich, St. Louis, MO, USA) containing a protease inhibitor cocktail and PhoSTOP (Roche Molecular Biochemicals, Basel, Switzerland). Ten micrograms of the protein lysate 
a

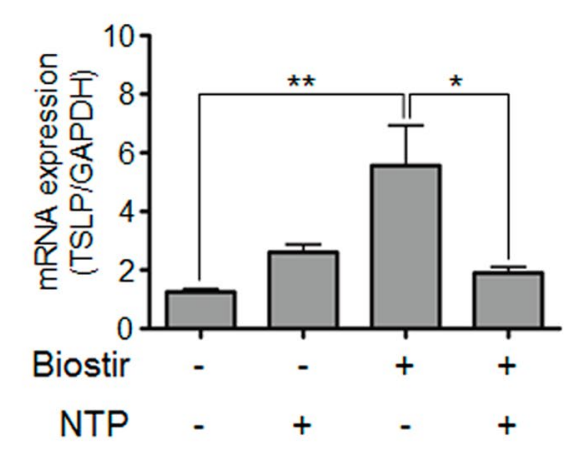

C
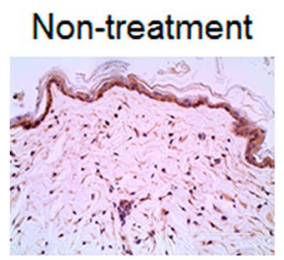

Biostir only

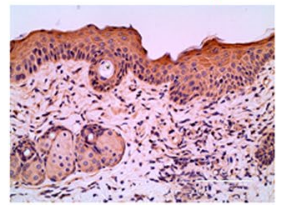

TSLP

NTP only
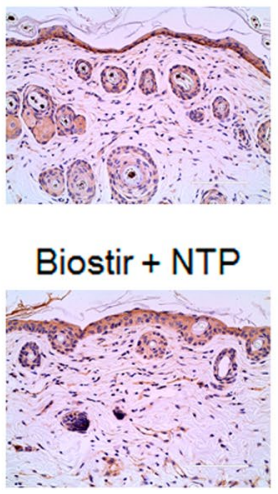

b

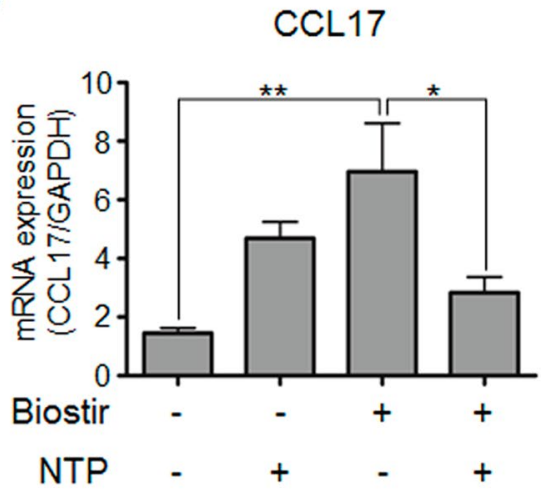

d

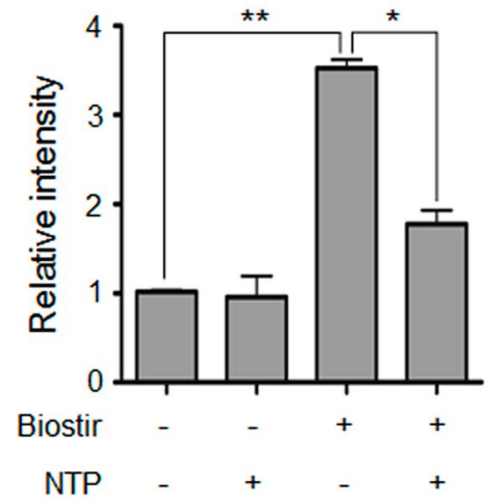

Figure 5. Cytokine and chemokine expression in the mouse skin. AD-related cytokine, (a) TSLP and (b) chemokine CCL17 expression increased in HDM-applied mouse skin and the expression was inhibited by NTP treatment. (c) NTP treatment reduced HDM-induced TSLP production in mouse skin. TSLP production was detected by IHC. (d) The level of TSLP is shown as a bar graph. $* P<0.05, * * P<0.01$. Bar $=100 \mu \mathrm{m}$.

were loaded into 10\% SDS-PAGE and transferred to PVDF membranes (Amersham, Arlington Heights, IL, USA). After transfer is finished, the membranes were incubated with blocking solution and then appropriate antibodies. The bands were visualized with Advanced ECL Western Blotting Detection Reagents (Amersham). GAPDH levels are used as a loading control.

Mice. Six-week-old male NC/Nga mice were obtained from Orient Bio (Korea). These mice were housed in a controlled room with a 12:12-hour light-dark cycle and free access to laboratory chow and water. Seven to nine-week-old NC/Nga mice were used for animal experiments. The animal experiments in this study were approved by the Committee for Ethics in Animal Experiment of Ajou University School of Medicine and performed in accordance with the institution guidelines.

HDM-induced AD in mice. AD was induced in NC/Nga mice by application of HDM as described previously with minor modification ${ }^{37}$. Briefly, dorsal hairs of the mice were removed completely. Next day, $200 \mu l$ of $4 \%$ SDS solution were used to dorsal skin to break the skin barrier followed by application of $100 \mathrm{mg}$ of HDM containing cream (Biostir) every 4 days for 3 weeks. All the mice were sacrificed at a day after the final treatment. The mice were divided into four groups: non-treated control, NTP-treated only, Biostir-treated only and the topical application of NTP to Biostir-treated mice.

Immunohistochemistry. The dorsal skins were fixed in $4 \%$ paraformaldehyde for $24 \mathrm{~h}$ and embedded in paraffin. The sections $(4.5 \mu \mathrm{m})$ were obtained, stained with $\mathrm{H} \& \mathrm{E}$ or toluidine blue to monitor histological changes in the skin and mast cell recruitment, respectively. Eosinophil peroxidase (EPX) staining was carried out using a goat polyclonal anti-EPX antibody (Santa Cruz Biotechnology) to determine the recruitment of eosinophils.

Mouse bone marrow-derived mast cells. BMMC were differentiated as previously described with minor changes ${ }^{38}$. Briefly, mouse bone marrow cells were collected from femurs of 7-8-week-old C57BL/6 mice and lineage-negative cells were purified using the lineage-negative cell isolation kit (Miltenyl Biotech, Germany) according to the manufacturer's suggested protocols. The lineage-negative cells were cultured in RPMI 1640 medium supplemented with $10 \% \mathrm{FBS}, 100 \mathrm{U} / \mathrm{ml}$ penicillin, and $100 \mu \mathrm{g} / \mathrm{ml}$ streptomycin in the presence of $10 \mathrm{ng} /$ $\mathrm{ml} \mathrm{mIL-3} \mathrm{(PeproTech,} \mathrm{USA)} \mathrm{and} 50 \mathrm{ng} / \mathrm{ml} \mathrm{mSCF}$ (PeproTech, USA) for eight weeks at $37^{\circ} \mathrm{C}$ in a humidified 
a
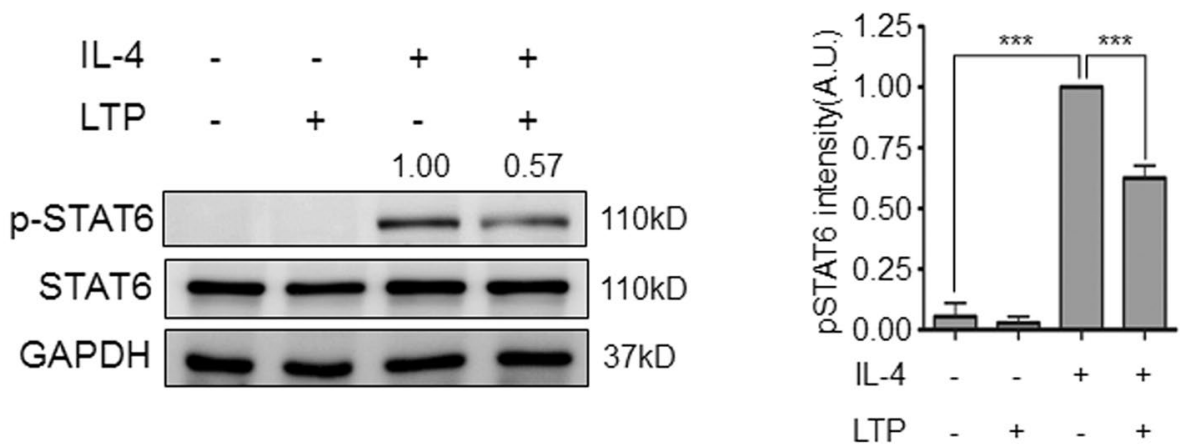

b

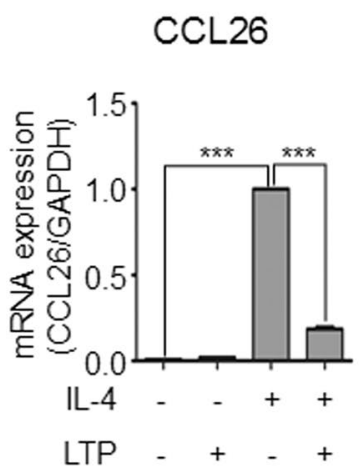

C TNF- $\alpha-+++$

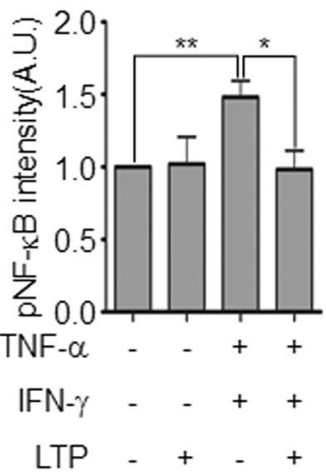

d

IL-8

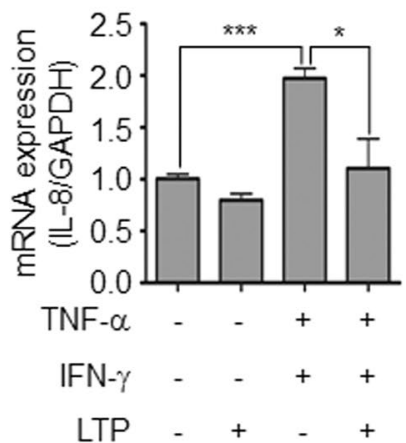

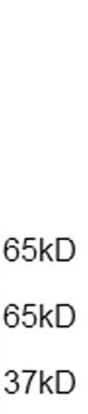

$\begin{array}{rllll}\text { IFN-Y } & - & - & + & + \\ \text { LTP } & - & + & - & +\end{array}$

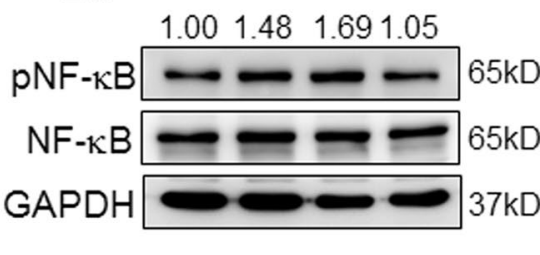

e

IL-6

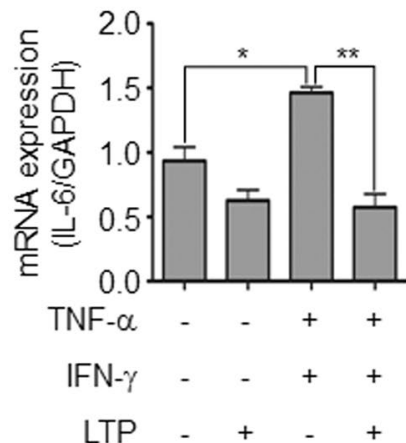

TNF- $\alpha$

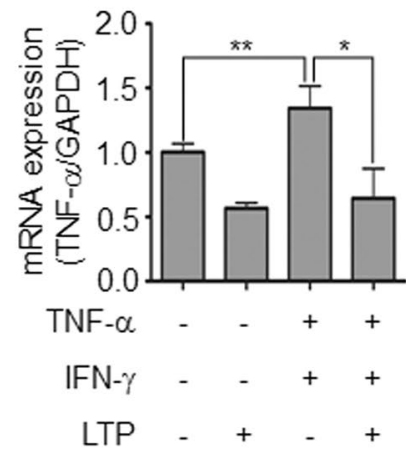

Figure 6. LTP treatment inhibits allergic inflammatory responses in HaCaT. (a) All Western blotting experiments were performed under the same condition. LTP treatment inhibited IL-4-induced STAT6 activation in HaCaT. IL-4 was stimulated with or without LTP for $15 \mathrm{~min}$ and the cells were collected for p-STAT6 detection using western blot analysis. The level of p-STAT6 was measured using ImageJ software. (b) LTP treatment inhibited IL-4-induced CCL26 expression in HaCaT. (c) All Western blotting experiments were performed under the same condition. LTP treatment inhibited TNF- $\alpha / \mathrm{IFN}-\gamma$-induced NF- $\kappa$ B activation in $\mathrm{HaCaT}$. HaCaT cells were stimulated with TNF- $\alpha$ and IFN- $\gamma$ for $15 \mathrm{~min}$ and the cells were harvested for

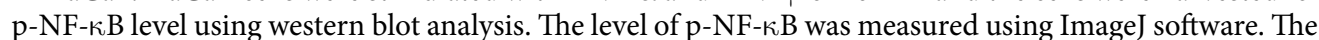
expression of pro-inflammatory chemokine, (d) IL-8, and cytokine, (e) IL-6 and (f) TNF- $\alpha$ was detected in TNF- $\alpha /$ IFN- $\gamma$-stimulated HaCaT with or without LTP treatment using real-time PCR analysis. $* P<0.05$, $* * P<0.01, * * * P<0.001$.

atmosphere with $5 \% \mathrm{CO}_{2}$. The mast cell differentiation was determined by FACS analysis, and $>94 \%$ of the cells were positive for CD117 and FceRI, which are mast cell markers.

Flow cytometry and intracellular cytokine analysis. Draining lymph nodes were isolated form mice and the lymph nodes were ground for single cell suspension. The cells were activated using anti-CD3 and anti-CD28 antibodies for 2 days and stained with anti-mouse IL-4, anti-mouse CD4 and anti-mouse CD3 antibodies (BioGems, Westlake Village, CA). Flow cytometry was performed on a FACSAria (BD Bioscience). 
Statistical analysis. Statistical comparisons between groups were performed using one-way analysis of variance (ANOVA) and Tukey's post hoc tests. $(*) P<0.05,(* *) P<0.01$ and $(* * *) P<0.001$ were considered significant. All experiments were performed at least three times.

\section{References}

1. Bieber, T. Atopic dermatitis. N. Engl. J. Med. 358, 1483-1494 (2008)

2. Leung, D. Y., Boguniewicz, M., Howell, M. D., Nomura, I. \& Hamid, Q. A. New insights into atopic dermatitis. J Clin Invest. 113, 651-657 (2004).

3. Leung, D. Y. Atopic dermatitis: new insights and opportunities for therapeutic intervention. J. Allergy Clin. Immunol. 105, 860-876 (2000).

4. Brandt, E. B. \& Sivaprasad, U. Th2 cytokines and atopic dermatitis. J Clin Cell Immunol. 2, 110 (2011).

5. Di Cesare, A., Di Meglio, P. \& Nestle, F. O. A role for Th17 cells in the immunopathogenesis of atopic dermatitis? J Invest Dermatol. 128, 2569-2571 (2008).

6. Novak, N. \& Bieber, T. Allergic and nonallergic forms of atopic diseases. J. Allergy Clin. Immunol. 112, 252-262 (2003).

7. Peng, W. \& Novak, N. Pathogenesis of atopic dermatitis. Clin Exp Allergy. 45, 566-574 (2015).

8. Misery, L. Therapeutic perspectives in atopic dermatitis. Clin Rev Allergy Immunol. 41, 267-271 (2010)

9. Berke, R., Singh, Q. \& Guralnick, M. Atopic dermatitis: an overview. Am Fam Physician 86, 35-42 (2012).

10. Kim, C. H. et al. Effects of atmospheric nonthermal plasma on invasion of colorectal cancer cells. Appl. Phys. Lett. 96, 243701-243703 (2010).

11. Kim, C. H. et al. Induction of cell growth arrest by atmospheric non-thermal plasma in colorectal cancer cells. Journal of Biotechnology 150, 530-538 (2010).

12. Partecke, L. et al. Tissue Tolerable Plasma (TTP) induces apoptosis in pancreatic cancer cells in vitro and in vivo. BMC Cancer 12, $473(2012)$.

13. Arndt, S. et al. Cold atmospheric plasma, a new strategy to induce senescence in melanoma cells. Experimental Dermatology 22, 284-289 (2013).

14. Emmert, S. et al. Atmospheric pressure plasma in dermatology: Ulcus treatment and much more. Clin Plasma Med. 1, 24-29 (2013).

15. Klampfl, T. G. et al. Cold atmospheric air plasma sterilization against spores and other microorganisms of clinical interest. Appl. Environ. Microbiol. 78, 5077-5082 (2012).

16. Park, J. K. et al. Feasibility of nonthermal atmospheric pressure plasma for intracoronal bleaching. Int Endodont J. 44, 170-175 (2011).

17. Kim, S. Y. et al. Non-thermal plasma induces AKT degradation through turn-on the MUL1 E3 ligase in head and neck cancer. Oncotarget 6, 33382-33396 (2015).

18. Choi, J. W. et al. Novel Therapeutic Effects of Non-thermal atmospheric pressure plasma for Muscle Regeneration and Differentiation. Sci. Rep. 6, 28829 (2016).

19. Kang, S. U. et al. N2 non-thermal atmospheric pressure plasma promotes wound healing in vitro and in vivo: Potential modulation of adhesion molecules and matrix metalloproteinase-9. Experimental dermatology 26, 163-170 (2017).

20. Lee, Y. S., Lee, M. H., Kim, H. J., Won, H. R. \& Kim, C. H. Non-thermal atmospheric plasma ameliorates imiquimod-induced psoriasis-like skin inflammation in mice through inhibition of immune responses and up-regulation of PD-L1 expression. Sci. Rep. 7, 15564 (2017).

21. Isbary, G. et al. A first prospective randomized controlled trial to decrease bacterial load using cold atmospheric argon plasma on chronic wounds in patients. British J. Dermatol. 163, 78-82 (2010).

22. Kang, S. U. et al. Nonthermal plasma induces head and neck cancer cell death: the potential involvement of mitogen-activated protein kinase-dependent mitochondrial reactive oxygen species. Cell Death Dis. 5, e1056 (2014).

23. Choi, J. H. et al. Skin renewal activity of non-thermal plasma through the activation of b-catenin in keratinocytes. Sci. Rep. 7, 6146 (2017).

24. Deckers, I. A. et al. Investigating international time trends in the incidence and prevalence of atopic eczema 1990-2010: a systematic review of epidemiological study. PLos One 7, e39803 (2012).

25. Heratizadeh, A. \& Werfel, T. Anti-inflammatory therapies in atopic dermatitis. Allergy 71, 1666-1675 (2016).

26. Coondoo, A., Phiske, M., Verma, S. \& Lahiri, K. Side-effects of topical steroids: a long overdue revisit. Indian Dermatol. Online J. 5, 416-425 (2014).

27. Furue, M. et al. Clinical dose and adverse effects of topical corticosteroids in daily management of atopic dermatitis. Br J Dermatol. 148, 128-133 (2003).

28. Kimura, M., Tsuruta, S. \& Yoshida, T. Correlation of house dust mite-specific lymphocyte proliferation with IL-5 production, eosinophilia, and the severity of symptoms in infants with atopic dermatitis. J Allergy Clin Immunol. 101, 84-89 (1998).

29. Tomimori, Y., Tanaka, Y., Goto, M. \& Fukuda, Y. Repeated topical challenge with chemical antigen elicits sustained dermatitis in NC/Nga mice in specific-pathogen-free condition. J Invest Dermatol. 124, 119-124 (2005).

30. Jin, H., He, R., Oyoshi, M. \& Geha, R. S. Animal models of atopic dermatitis. J Invest Dermatol. 129, 31-40 (2009).

31. Kim, S. Y. et al. Transduced PEP-1-FK506BP ameliorates atopic dermatitis in NC/Nga mice. J Invest Dermatol. 131, 1477-1485 (2011).

32. Lunov, O. et al. Chemically different non-thermal plasmas target distinct cell death pathways. Sci. Rep. 7, 600 (2017).

33. Smolkova, B. et al. Non-thermal plasma, as a new physicochemical source, to induce redox imbalance and subsequent cell death in liver cancer cell lines. Cell. Physiol. Biochem. 52, 119-140 (2019).

34. Raza, H. M. et al. ROS-modulated therapeutic approaches in cancer treatment. J. Cancer Res. Clin. Oncol. 143, 1789-1809 (2017).

35. Choi, J. H., Song, Y. S., Lee, H. J., Hong, J. W. \& Kim, G. C. Inhibition of inflammatory reactions in 2,4-dinitrochlorobenzene induced NC/Nga atopic dermatitis mice by non-thermal plasma. Sci. Rep. 6, 27376 (2016).

36. Shin, Y. S. et al. Anti-cFancer Effect of Luminacin, a Marine Microbial Extract, in Head and Neck Squamous Cell Carcinoma Progression via Autophagic Cell Death. Cancer Res Treat 48, 738-752 (2016).

37. Yamamoto, M. et al. A novel atopic dermatitis model induced by topical application with dermatophagoides farinae extract in NCNga mice. Allergol Int 56, 139-148 (2007).

38. Lee, J. H., Lee, Y. S., Lee, E. J., Lee, J. H. \& Kim, T. Y. Capsiate inhibits DNFB-induced atopic dermatitis in NC/Nga mice through mast cell and CD4+ T cell activation. J Invest Dermatol. 135, 1977-1985 (2015).

\section{Acknowledgements}

This study was supported by the Basic Science Research Program (2017M3A9F7079339, 2011-0030034, and 2018R1A2B3009008) through the National Research Foundation of Korea funded by the Ministry of Science, ICT, and Future Planning (MSIP). 


\section{Author Contributions}

Y.S.L., S.U.K., M.-H.L. and C.H.K. analyzed the data. Y.S.L. and C.H.K. wrote the manuscript. M.-H.L., Y.S.L., C.H.K. and H.J.K. performed the experiments. M.-H.L. drew schematic diagram. All authors reviewed the manuscript.

\section{Additional Information}

Supplementary information accompanies this paper at https://doi.org/10.1038/s41598-019-49938-9.

Competing Interests: The authors declare no competing interests.

Publisher's note Springer Nature remains neutral with regard to jurisdictional claims in published maps and institutional affiliations.

(c) (i) Open Access This article is licensed under a Creative Commons Attribution 4.0 International License, which permits use, sharing, adaptation, distribution and reproduction in any medium or format, as long as you give appropriate credit to the original author(s) and the source, provide a link to the Creative Commons license, and indicate if changes were made. The images or other third party material in this article are included in the article's Creative Commons license, unless indicated otherwise in a credit line to the material. If material is not included in the article's Creative Commons license and your intended use is not permitted by statutory regulation or exceeds the permitted use, you will need to obtain permission directly from the copyright holder. To view a copy of this license, visit http://creativecommons.org/licenses/by/4.0/.

(C) The Author(s) 2019 